Tropical Journal of Pharmaceutical Research May 2017; 16 (5): 1167-1172

ISSN: $1596-5996$ (print); 1596-9827 (electronic)

(C) Pharmacotherapy Group, Faculty of Pharmacy, University of Benin, Benin City, 300001 Nigeria.

All rights reserved.

Available online at http://www.tjpr.org

Original Research Article

http://dx.doi.org/10.4314/tjpr.v16i5.26

\title{
Eosinophilia triggers changes in IL-5, eotaxin and IL-17, and acts as a prognostic biomarker for atopic dermatitis
}

\author{
Zhi-hong $\mathrm{Wu}^{*}$, Jiang Zhong, Chuan-li Su, Yun-shu Huang, Tao Huang and \\ Zhang-jie Xu \\ Department of Dermatology, First Affiliated Hospital, Guangxi University of Chinese Medicine, Nanning 530023, China
}

*For correspondence: Email: wuzhihongdoc@sina.com; Tel: +86-0771-5848587

Sent for review: 4 August 2016

Revised accepted: 6 April 2017

\begin{abstract}
Purpose: To investigate the implication of eosinophilia in atopic dermatitis $(A D)$.

Methods: A total of 139 AD patients from The First Affiliated Hospital of Guangxi University of Chinese Medicine between February 2013 and May 2015, were involved in this study. Scoring atopic dermatitis (SCORAD) index was used to evaluate the skin lesions. The levels of IL-4, IL-5, IL-13, IL-17, INFgamma, IP-10, eotaxin and Regulated on Activation, Normal T-cell Expressed and Secreted (RANTES), were determined with commercial enzyme-linked immunosorbent assay (ELISA) kits. Eosinophil counts were carried out by granulocyte count method. Correlation between SCORAD scores and levels of cytokines was analyzed using the Spearman correlation method.

Results: SCORAD scores significantly increased in the eosinophil-positive group when compared to eosinophil-negative group $(p<0.05)$. Eosinophil counts correlated with SCORAD scores in the eosinophil-positive group $(p<0.05)$. INF-y, IP-10 and RANTES levels were significantly higher in the eosinophil-positive group than in eosinophil- negative group, while IL-5, eotaxin and IL-17 levels significantly decreased in eosinophil-positive group $(p<0.05)$. In the eosinophil-positive group, IL-5, eotaxin and IL-17 levels positively correlated with SCORAD scores.

Conclusion: Eosinophilia triggers IL-5, eotaxin and IL-17 changes and acts as a prognostic biomarker for atopic dermatitis. These findings may give further insights into the pathogenesis of $A D$.
\end{abstract}

Keywords: Atopic dermatitis, Eosinophilia, SCORAD score, Biomarker, Cytokines

Tropical Journal of Pharmaceutical Research is indexed by Science Citation Index (SciSearch), Scopus, International Pharmaceutical Abstract, Chemical Abstracts, Embase, Index Copernicus, EBSCO, African Index Medicus, JournalSeek, Journal Citation Reports/Science Edition, Directory of Open Access Journals (DOAJ), African Journal Online, Bioline International, Open-J-Gate and Pharmacy Abstracts

\section{INTRODUCTION}

Atopic dermatitis (AD) is a common chronic inflammatory and relapsing skin disease, which is always associated with food allergies and asthma [1]. Previous studies showed that industrialization and urbanization influence the rates of atopic disease [2,3]. Recent studies revealed that the prevalence of $A D$ decreases from in central and rural areas of Shanghai, China [4]. The International Study of Asthma and Allergies in Childhood (ISAAC) showed that the incidence and prevalence of $A D$ are on the increase all over the world, especially in the developing countries [5]. AD places a heavy economic burden on the society and is a source of huge financial costs to health systems [6].

Many studies have been reported that $A D$ may be induced when the skin is stimulated by allergens $[7,8]$. Recent studies also reported that some of environmental allergens could produce eczematous reactions on the skin $[7,8]$. However the pathogenesis of $A D$ and its immune responses are still not clearly understood. It has been shown that chronic immune activation 
contributes to the pathophysiology of this common skin disease, and that eosinophils are frequently observed in $A D$ patients [8].

Elevated blood eosinophil count is an important characteristic of $A D$ patients [9]. However, the reasons for the elevation in blood eosinophil count have not been fully elucidated [10]. It has been speculated that the eosinophils may play protective roles in AD $[9,10]$. Previous studies have demonstrated that eosinophils are activated pro-inflammatory cells which could cause some of the allergic inflammation symptoms.

Recent studies on activities of eosinophils revealed that they contain potent toxic proteins with the potential to mediate tissue damage [810]. Furthermore, immuno-fluorescent localization of eosinophil granule proteins has shown that the toxic granule proteins were deposited in tissues when the eosinophils become disrupted [10]. The deposition of granule proteins in several diseases is vastly out of proportion with the number of identifiable cells [9]. Specifically, the deposition of eosinophil granule proteins outside of eosinophils has been observed in lichenified eczematous disorders with elevated serum levels of immunoglobulin E; in urticarial and angioedematous disorders, and in bullous diseases [10].

The present study was carried out to investigate the prognostic biomarker potential of eosinophils in $A D$ patients.
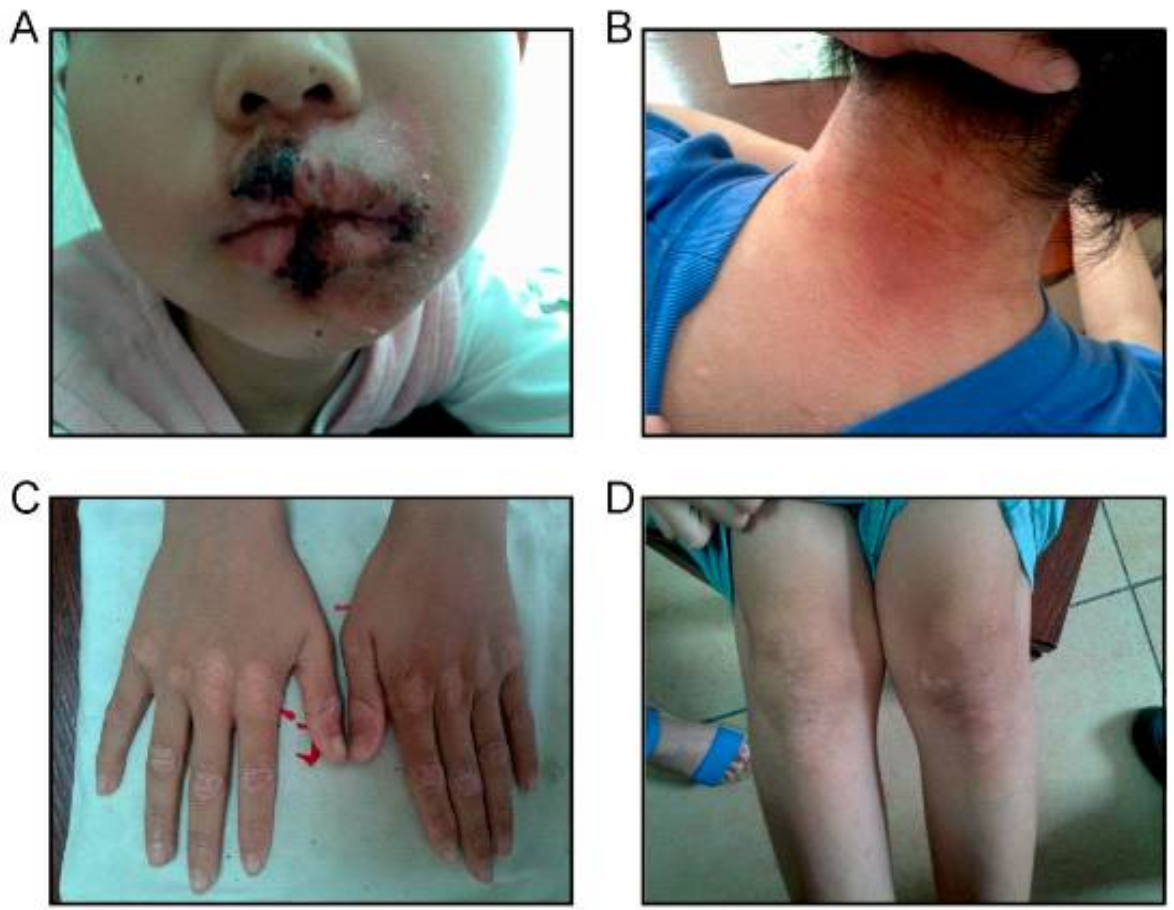

Figure 1: Patients clinically diagnosed with AD illustrating different syndromes. A. Dermatitis at oral lips. B. Dermatitis at nape. C. Dermatitis at hand. D. Dermatitis at leg

\section{EXPERIMENTAL}

\section{Patients}

The survey was performed at the First Affiliated Hospital of Guangxi University of Chinese Medicine, China. A total of 139 children aged 2 to 12 years, who were clinically diagnosed with $A D$, were recruited. This study was approved by the Ethical Committee of The First Affiliated Hospital of Guangxi University of Chinese Medicine, Nanning, China (approval no. [2013]-K002). Informed consents were obtained from all of the participants. All of the patients have been approved this study. The duration of disease in every patient was more than six months, and the remission was less than 3 months in a year (Figure 1). In accordance with the Guidelines of the World Medical Association Declaration of Helsinki [11], patients were treated with loratadine granules per os and desonide for external use.

All the $A D$ patients underwent 2 year-long followup and 3 months of regular visits. Blood samples were collected for determination of eosinophil counts and related cytokines, including IL-4, IL-5, IL-13, IL-17, INF-gamma, IP-10, eosinophil chemotactic factor eotaxin and RANTES. The study subjects were grouped into eosinophilpositive group (58 cases) and eosinophilnegative group (61 cases). 


\section{ELISA and eosinophil counts assay}

Blood levels of IL-4, IL-5, IL-13, IL-17, INFgamma, IP-10, eotaxin and RANTES were determined using ELISA commercial kits. Eosinophil counts were carried out using granulocyte count method.

\section{European AD score (SCORAD)}

Scoring atopic dermatitis index (SCORAD) was used as $A D$ standard to assess the extent of skin lesions (A), the severity of skin lesions (B) and pruritus and sleep $(C)$. The score guide was as follows:

A (1) for adults: $9 \%$ for head, neck and arm; $13.5 \%$ for each side of trunk, and $22.5 \%$ for the lower extremities. A (2) for children under 14 years of age: $9 \%$ for head and neck, and $18 \%$ for arm, trunk and lower limbs.

B represented scores $(0-3)$ in respect skin lesions such as erythema, papules, edema, skin exfoliation, cracking, chapping and exudation, scab, moss or dry skin. C, which represented scores $(0-3)$ for pruritis and extent of effect on sleep, was divided into four grades: severe (3 points), moderate (2 points), mild (1 point) and nil (0 point).

\section{Statistical analysis}

Data analyses were performed using SPSS 20.0 software. Student's $t$ test was used to evaluate significant differences between groups. $P<0.05$ was considered statistically significant. Correlation between SCORAD scores and levels of cytokines were analyzed using Spearman correlation method.

\section{RESULTS}

\section{SCORAD scores}

The results indicated that eosinophil counts in the eosinophil-positive group were significantly higher when compared to corresponding values in the eosinophil-negative group, irrespective of AD duration $(p<0.05$, Table 1$)$. SCORAD scores were also significantly higher in the eosinophilpositive group than in the eosinophil-negative group in "Half year", "One year", "One-and-half year" and "Two years" categories $(p<0.05$, Table 1). However, eosinophil counts and SCORAD scores were significantly lowered from "Half year" to "Two years" in the eosinophilnegative group $(p<0.05$, Table 1$)$. SCORAD scores were correlated with eosinophil counts in both groups. The results also revealed that eosinophil counts were positively correlated with SCORAD scores in the eosinophil-positive group (Figure 2, $p<0.05$ ).
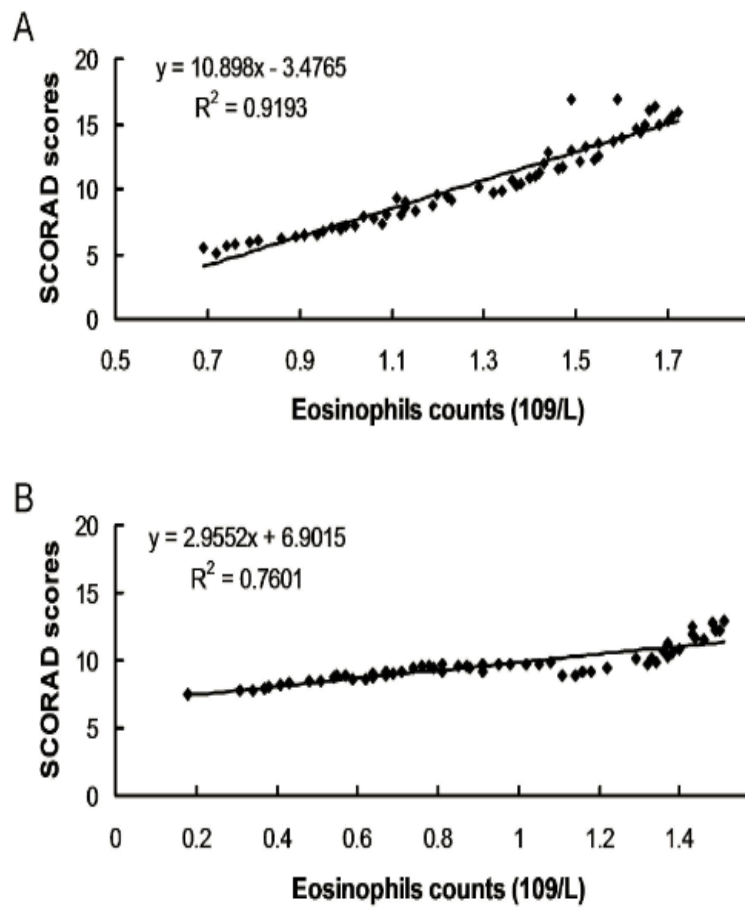

Figure 2: Correlation analysis between SCORAD scores and the eosinophils counts in eosinophils positive group $(A)$ and negative group $(B)$

\section{INF- $y$, IP-10 and RANTES levels}

The results showed that INF-y, IP-10 and RANTES levels were significantly higher in "One year" compared to "Half year"; and in "One-andhalf year" $A D$ when compared to "One year" $A D$ (post-diagnosis) in the eosinophil-positive group $(p<0.05$, Table 2). For the INF-y and RANTES,

Table 1: Eosinophil counts and SCORAD scores in eosinophils positive and negative groups at half year, one year, one and half year and two years after diagnosis of $A D$

\begin{tabular}{lcccc}
\hline Time & \multicolumn{2}{c}{ Eosinophils positive group } & \multicolumn{2}{c}{ Eosinophils negative group } \\
\cline { 2 - 4 } & Counts (109/L) & SCORAD & Counts (109/L) & SCORAD \\
\cline { 2 - 4 } Half year & $0.97 \pm 0.28$ & $6.28 \pm 1.77$ & $1.32 \pm 0.29$ & $10.05 \pm 3.12$ \\
One year & $1.05 \pm 0.33$ & $9.41 \pm 2.85$ & $1.06 \pm 0.25$ & $6.91 \pm 1.83$ \\
One and half year & $1.53 \pm 0.41$ & $14.03 \pm 4.26$ & $1.06 \pm 0.25$ & $9.24 \pm 3.07$ \\
Two year & $1.46 \pm 0.37$ & $15.74 \pm 5.14$ & $0.47 \pm 0.08$ & $8.32 \pm 2.75$ \\
\hline
\end{tabular}


Table 2: Observation for the INF- $y$, IP-10 and RANTES expression in the eosinophils positive group at half year, one year, one and half year and two years after diagnosis of $A D$

\begin{tabular}{lccc}
\hline Time & INF-y $(\mathbf{p g} / \mathbf{m l})$ & IP-10 $(\mathbf{p g} / \mathbf{m l})$ & RANTES $(\mathbf{p g} / \mathbf{m L})$ \\
\hline Half-year & $29.563 \pm 3.815$ & $70.324 \pm 43.501$ & $54.813 \pm 12.068$ \\
One year & $47.06 \pm 5.493$ & $116.550 \pm 58.947$ & $93.054 \pm 30.245$ \\
One and half year & $324.612 \pm 76.524$ & $209.548 \pm 97.513$ & $912.356 \pm 105.436$ \\
Two years & $291.64 \pm 64.307$ & $241.538 \pm 122.095$ & $876.590 \pm 94.021$ \\
\hline
\end{tabular}

Table 3: Examination of the IL-5, extaxin and IL-17 expression in the eosinophils positive group at half year, one year, one and half year and two years after diagnosis of $A D$

\begin{tabular}{lccc}
\hline Time & IL-5 $(\mathbf{p g} / \mathbf{m L})$ & Eotaxin $(\mathbf{p g} / \mathbf{m L})$ & IL-17 $(\mathbf{p g} / \mathbf{m L})$ \\
\hline Half year & $84.786 \pm 10.257$ & $54.910 \pm 20.136$ & $92.744 \pm 17.391$ \\
One year & $75.042 \pm 9.035$ & $34.756 \pm 15.382$ & $30.927 \pm 3.895$ \\
One and half year & $66.793 \pm 8.578$ & $49.651 \pm 18.523$ & $75.782 \pm 8.653$ \\
Two year & $36.245 \pm 5.749$ & $41.207 \pm 16.113$ & $58.237 \pm 6.507$ \\
\hline
\end{tabular}
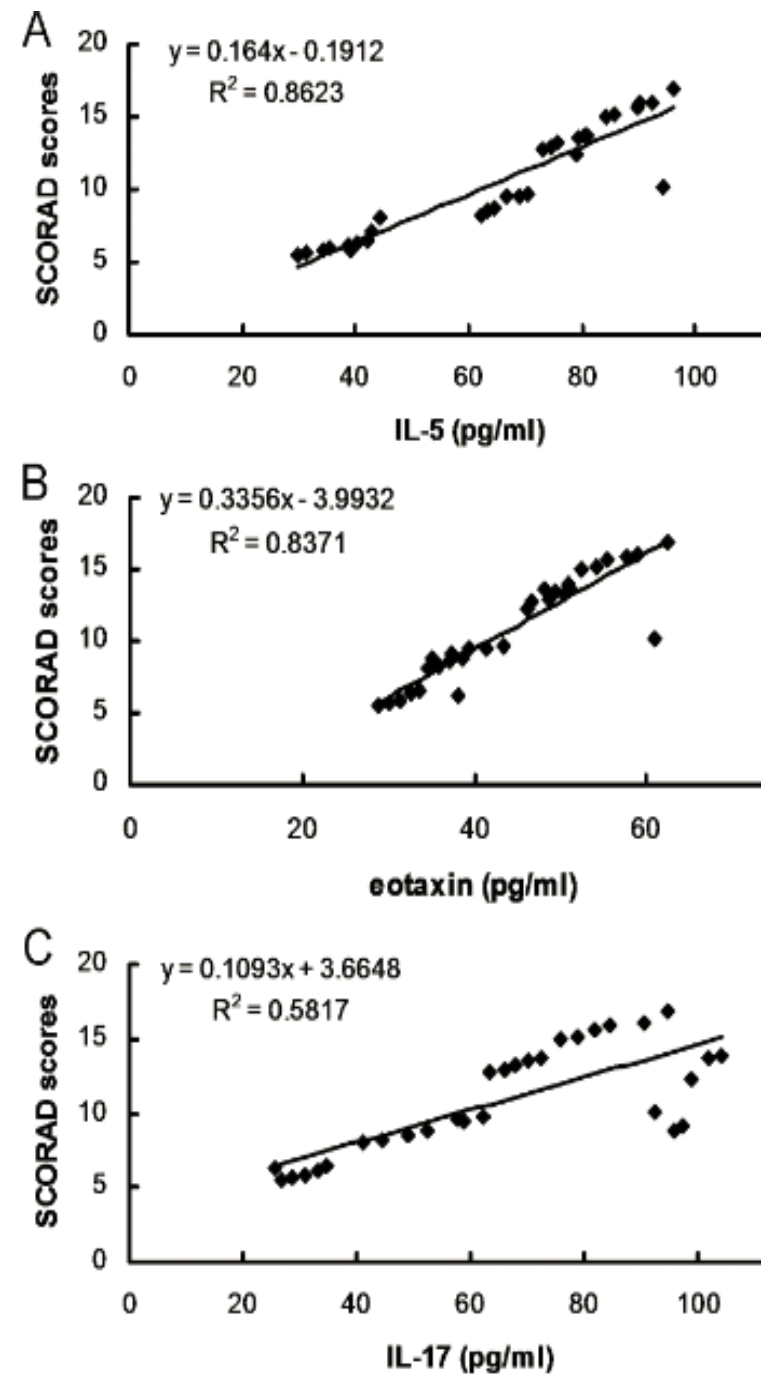

Figure 3: Correlation analysis between SOCRAD scores and the INF-Y (A), IP-10 (B) and RANTES (C) levels in the ensinophils positive group

the levels were significantly lower in the "Two years" compared to "One-and-half year" AD post-diagnosis (Table 2).
Results from analysis of the correlation between SCORAD scores and INF- $\mathrm{y}, \mathrm{IP}-10$ and RANTES levels indicated that there were no significant correlations between INF- $y$ and SCORAD scores (Figure $3 \mathrm{~A}, p>0.05$ ); or between $\mathrm{IP}-10$ and SCORAD scores (Figure 3B,) or between RANTES and SCORAD scores (Plate 3C, $p>$ 0.05).

\section{IL-5, eotaxin and IL-17 levels}

The results obtained showed that the levels of IL5 , eotaxin and $\mathrm{IL}-17$ were significantly lower in "One year", "One-and-half year" and "Two-year" post AD diagnosis categories when compared to the "Half year" post-diagnosis group (Table $3, p$ $<0.05$ ).

Results from cytokine assays showed that IL-5, extaxin and IL-17 levels were positively correlated with SCORAD scores (Figure 4, $p<0.05)$.

Furthermore, IL-4 and IL-13 levels were significantly lower in "One year", "One-and-half year" and "Two-year" when compared to "Half year" post-diagnosis (data not shown). However, IL-4 and IL-13 were not correlated with SCORAD scores in the eosinophil-positive patients (data not shown).

negative groups. The results indicated that SCORAD scores were significantly increased in the eosinophil-positive group. Furthermore, eosinophil counts correlated positively with SCORAD scores in eosinophil-positive group. These results suggest that eosinophil levels were associated with the SCORAD scores in the eosinophil-positive patients, indicating that eosinophil levels could reflect the level of skin injury in $A D$ patients. 

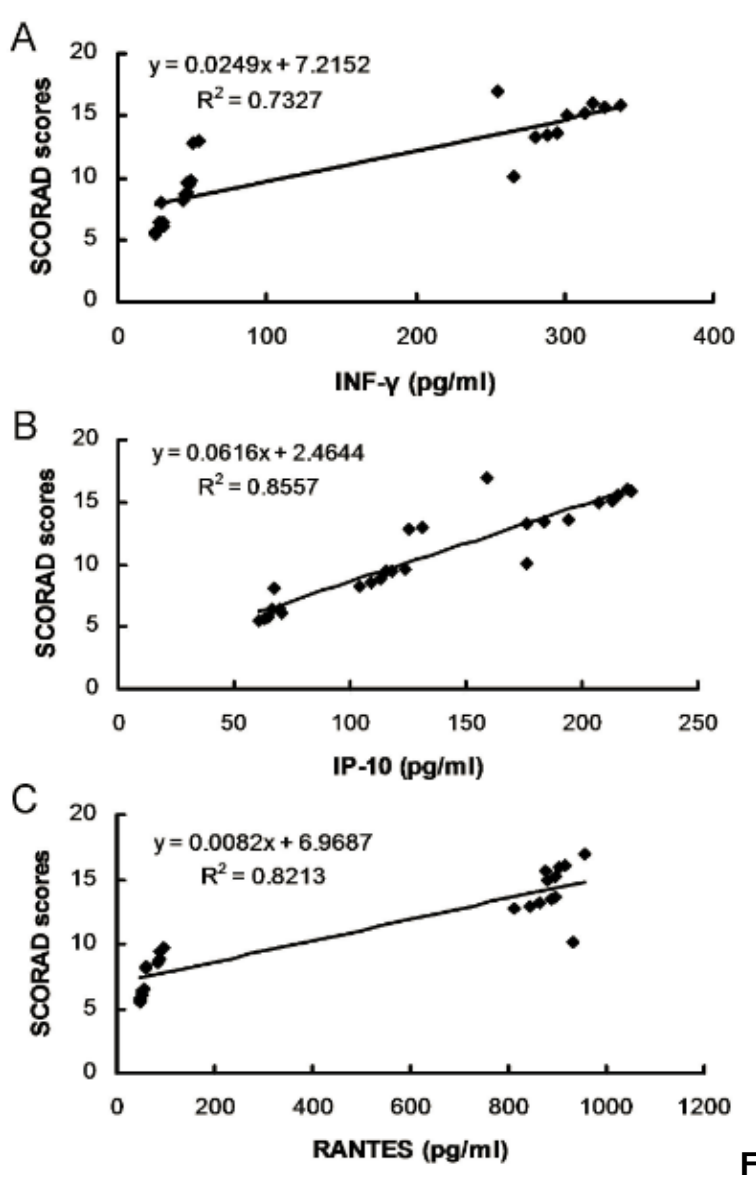

igure 4: Correlation analysis between SOCRAD scores and the IL-5 (A), eotaxin (B) and IL-17 (C) levels in the eosinophils positive group

Although INF- $\mathrm{y}, \mathrm{IP}-10$ and RANTES levels were significantly increased in the eosinophil-positive group, no correlations were established between levels of INF-y, IP-10 and RANTES, and SCORAD scores in the eosinophil-positive patients. These results suggest that the INF-y, IP-10 and RANTES cannot be used to evaluate the severity of AD. However, IL-5, eotaxin and IL17 levels were correlated with SCORAD scores in eosinophil-positive patients, indicating that the IL-5, eotaxin and IL-17 can be used to evaluate the severity of AD. Studies by Valirlis et al [17] also found that some of the cytokine were associated with SCORAD scores in acute $A D$ patients. However, not much was known about the involvement of INF-y, IP-10, RANTES, IL-5, eotaxin and IL-17. The present study is the first report to demonstrate that cytokines, IL-5, eotaxin and IL-17 levels correlated with SCORAN scores and skin injury in eosinophilpositive patients. However, IL-4 and IL-13 were not of predictive significance for the prognosis of $A D$.

\section{DISCUSSION}

$A D$ is a chronic, relapsing and highly pruritic dermatitis which always develops in early childhood. It has a characteristic age-dependent distribution. About 10 to $20 \%$ children suffer from $A D$ in the developing countries [12]. $A D$ is characterized by sino-pulmonary infections, dermatitis, cutaneous viral infections, altered eosinophil levels, elevated serum IgE, squamous cell carcinomas, and a high incidence of food allergies [12-14]. Aarkawa et al reported that different cytokines are present in the peripheral blood mononuclear cells of patients with atopic dermatitis [15]. Therefore, in this study, we investigated the prognostic value of eosinophil and related cytokines in atopic dermatitis.

Previous studies reported that the peripheral blood eosinophil could act as a diagnostic parameter in differentiating allergic $A D$ from nonallergic AD [16]. The present study also showed that eosinophil counts in $A D$ patients changed significantly, which is consistent with the previous study [16]. Furthermore, the AD patients were divided into eosinophil-positive and eosinophil-

\section{CONCLUSION}

The results obtained in this study strongly suggest that eosinophils act as prognostic biomarkers for $A D$ by triggering changes in IL-5, eotaxin and IL-17. The findings of this study may give further insights into the pathogenesis of AD.

\section{DECLARATIONS}

\section{Acknowledgement}

None declared

\section{Conflict of Interest}

No conflict of interest associated with this work.

\section{Contribution of Authors}

The authors declare that this work was done by the authors named in this article and all liabilities pertaining to claims relating to the content of this article will be borne by them.

\section{Open Access}

This is an Open Access article that uses a funding model which does not charge readers or their institutions for access and distributed under the terms of the Creative Commons Attribution License (http://creativecommons.org/licenses/by/ 4.0) and the Budapest Open Access Initiative (http://www.budapestopenaccessinitiative.org/rea d), which permit unrestricted use, distribution, 
and reproduction in any medium, provided the original work is properly credited.

\section{REFERENCES}

1. Boguniewicz M, Leung DYM. Atopic dermatitis: A disease of altered skin barrier and immune dysregulation. Immunol Rev 2011; 242(1): 233-246.

2. Mercer MJ, Joubert G, Ehrlich RI, Nelson H, Poyser MA, Puterman A, Weinberg EG. Socioeconomic status and prevalence of allergic rhinitis and atopic eczema symptoms in young adolescents. Pediatr Allergy Immunol 2004; 15(3): 234-241.

3. Yemaneberhan H, Bekele Z, Venn A, Lewis S, Parry E, Britton J. Prevalence of wheeze and asthma and relation to atopy in urban and rural Ethiopia. Lancet 1997; 350(1): 85-90.

4. Xu F, Yan S, Li F, Cai M, Wu M, Fu C, Zhao Z, Kan H, Kang $K, X u$ J. Prevalence of childhood atopic dermatitis: an urban and rural community-based study in Shanghai, China. PLoS One 2012; 7(5): e36174.

5. Asher MI, Montefort S, Bjorksten B, Lai CK, Strachan DP, Weiland SK, Willams H, ISSAC Phase Three Study Group. Worldwide time trends in the prevalence of symptoms of asthma, allergic rhinoconjunctivitis, and eczema in childhood: ISAAC phase one and three repeat multicountrycross-sectional surveys. Lancet 2006; 368(9537): 733-743.

6. Niwa $Y$, Sumi H, Kawahira K, Terashima T, Nakamura $T$, Akamatsu $\mathrm{H}$. Protein oxidative damage in the stratum corneum: Evidence for a link between environmental oxidants and the changing prevalence and nature of atopic dermatitis in Japan. Br J Dermatol 2003; 149(2): 248-254.

7. Bruynzeel-Koomen CA, Van Wichen DF, Spry CJ, Venge $P$, Bruynzeel P. Active participation of eosinophils in patch test reactions to inhalant allergens inpatients with atopic dermatitis. Br J Dermatol 1988; 118(2): 229-238.

8. Gondo A, Saeki N, Tokuda Y. Challenge reactions in atopic dermatitis after percutaneous entry of mite antigen. Br J Dermatol 1986; 115(4): 485-493.
9. Rajka G. Essential aspects of atopie eczema. Berlin: Springer, 1989

10. Leiferman KM. A current perspective on the role of eosinophiisin dermatologic diseases. J Am Acad Dermatol, 1991; 24(6Pt2): 1101-1112.

11. World Medical Association. World Medical Association Declaration of Helsinki: ethical principles for medical research involving human subjects. JAMA 2013; 310(20): 2191-2194.

12. Williams $H$, Robertson $C$, Stewart A, Ait-Khaled $N$, Anabwani G, Anderson R, Asher I, Beasley R, Bjorksten $B$, Turr M, Clayton T, Crane. Worldwide variations in the prevalence of symptoms of atopic eczema in the International Study of Asthma and Allergies in Childhood. J Allergy Clin Immunol 1999; 103(1): 125138.

13. Sanal $O$, Jing $H$, Ozgur $T$, Ayvaz D, Strauss-Albee DM, Ersoy-Evans S, Tezcan I, Turkkani G, Matthews HF, Haliloglu G, Yuce A, et al. Additional diverse findings expand the clinical presentation of DOCK 8 deficiency. $J$ Clinlmmunol 2012; 32(4): 698-708.

14. Su HC. Dedicator of cytokinesis 8 (DOCK8) deficiency. Curr Opin Allergy Clin Immunol 2010; 10(6): 515-520.

15. Arakawa $S$, Hatano $Y$, Katagiri K. Differential expression of mRNA for Th1 and Th2 cytokine-associated transcription factors and suppressors of cytokine signaling in peripheral blood mononuclear cells of patients with atopic dermatitis. Clin Exp Immunol 2004; 135(3): 505-510.

16. Jenerowicz $D$, Czarnecka-Operacz $M$, Silny $W$. Peripheral blood eosinophilia in atopic dermatitis. Acta Dermatovenerol Alp Pannonica Adriat2007; 16(1): 4752.

17. Vakirlis E, Lazaridou E, Tzellos TG, Gerou S, Chatzidimitriou $D$, loannides $D$. Investigation of cytokine levels and their association with SCORAD index in adults with acute atopic dermatitis. J Eur Acad Dermatol Venereol 2011; 25(4): 409-416. 